\title{
Content-Based Efficient Messages Transmission in WSNs
}

\author{
Rong Xiao, ${ }^{1}$ Bo Sun $\mathbb{D D}^{1},{ }^{1}$ Yongkang Xiao, ${ }^{1}$ Yungang Wei, ${ }^{1}$ and Wenlong Chen $\mathbb{D}^{2}$ \\ ${ }^{1}$ College of Information Science and Technology, Beijing Normal University, Beijing 100875, China \\ ${ }^{2}$ College of Information Engineering, Capital Normal University, Beijing 100048, China \\ Correspondence should be addressed to Bo Sun; tosunbo@bnu.edu.cn
}

Received 23 March 2018; Revised 23 June 2018; Accepted 30 July 2018; Published 13 August 2018

Academic Editor: Dongkyun Kim

Copyright (C) 2018 Rong Xiao et al. This is an open access article distributed under the Creative Commons Attribution License, which permits unrestricted use, distribution, and reproduction in any medium, provided the original work is properly cited.

\begin{abstract}
The WSNs are mainly to monitor various types of sensing content. In the automated control system, administration centers (ACs) often send notifications to a set of nodes meeting given content ranges to implement specific actions. For instance, we notify the sensing devices with sensing temperatures greater than 35 to turn on the cooling system. At present, the transmission of notification messages is mostly based on node identification, which is isolated from sensing contents and unable to accurately locate nodes meeting the sensing content requirements. In this paper, we generalize two types of sensing contents: the continuous values and the discrete values, and a content-based efficient message transmission mechanism (CEMT) is proposed for the typical tree-like topologies in WSNs. Thus, a highly effective notifying method for content-based multicast and anycast messages is designed, which accurately sends notification messages to nodes whose sensing values belong to the given range. Sometimes, multiple sensing types of nodes are mixed together to construct a net topology, offering underlying transport services to each other. At this point, CEMT builds a specialized logical tree for the same type of nodes, and notification messages are transmitted along the logical tree. CEMT reduces the transmission time, bandwidth, and number of processing nodes when a content-based message is sent, and it takes less storage of content routing entries in nodes.
\end{abstract}

\section{Introduction}

Current routing mechanisms in WSNs are implemented mainly based on node identities. For instance, the ROLL [1] workgroup designed the Routing Protocol for low-power and lossy network (RPL) [2], which fulfills routing in light of IP addresses in tree-like topologies. However, this kind of routing mechanism only provides the transmission reachability between wireless nodes with multiple hops. At the same time, most current studies on WSNs focus on data transmission from sensor nodes to the sink node, which mainly completes the collection of sensing content. Actually, for the automatic remote control in WSNs, efficient transmission from the sink nodes to sensor nodes is also an important part. In our previous research [3], we briefly introduced a notification transmission mechanism based on sensing content. In this paper, we will do further research work, especially on the common networking of multitype sensor nodes, performance analysis on storage consumption and transmission delay, and so on.
As we know, WSNs are often deployed to monitor specific environment parameters, and the communications in WSNs always resolve around sensor contents. Therefore, in this paper, we mainly study how to improve the data transmission performance from the sink to sensor nodes based on the sensing content.

In this paper, we assume that messages are sent to some nodes whose sensing contents meet certain conditions. For example, administration centers (ACs) need to inform the nodes that the temperatures are greater than 35 to take some actions. Nevertheless, in general, ACs are unware of the number and IP addresses of the nodes that satisfies the sensing content requirement. Therefore, ACs could only send notifications to all nodes, and the receiving nodes judge whether to deal with the received messages or not. The above message is a kind of multicast message, which is sent a set of nodes meeting the requirement. In addition, there is an anycast message in this paper, which only needs to be sent to one node that meets the requirements. Even if there are multiple nodes in the topology that meet the requirements, 
we just need to make sure that one node that meets the criteria receives the message.

Current routing mechanisms based on node identities fail to provide a high-performance transmission for notification messages with sensor contents. Our works focus on 2 aspects: (1) How to improve the completion speed of message transmission; (2) How to reduce the resource cost of messages transmission like bandwidth and processing. Targeting at the above objectives, we propose a contentbased efficient messages transmission mechanism (CEMT) in WSNs. Firstly, two kinds of sensor contents are summarized, namely, the continuous sensor contents and the discrete sensor contents. We design a storage method for content values which serves for the above two kinds of sensor contents at the same time. In tree topologies, sensing contents are aggregated at the root node of each subtree. Then, the message transmission modes are summarized including the multicast message notification and the anycast message notification, and an efficient transmission mechanism for these two modes, which can efficiently send the notification messages carrying content rules to the nodes that meet the rules requirements. Furthermore, in real deployments, sometimes there are several different types of sensor nodes in one subnet. Due to this circumstance, we construct a logical tree for each kind of sensing content, and a notification message is sent to the destination nodes along its logical tree.

The remainder of the paper is organized as follows. Section 2 introduces the related work. In Section 3, the problems faced by content-based messages transmission are introduced through a specific example. At the same time, some related definitions and the notification transmission mechanism based on sensing content are presented. Then, in Section 4, we give the detailed description of the transmission mechanism and the algorithms involved, including sensing contents aggregation and messages procession. Section 5 introduces how the transmission mechanism is implemented in the subnet with multidimensioned sensing contents. Section 6 presents the experiments and correlative performance analysis. Finally, the conclusion is drawn in Section 7.

\section{Related Work}

Contents based addressing and routing have been paid much attention in the academic circle $[4,5]$. For instance, [5] proposed an adaptive publish-subscribe distance vector protocol, which can provide efficient routing transmission based on distance vector for the named data objects. In order to improve the routing efficiency, an embedded multilevel ring (MVR) structure was proposed [6], which identifies senor nodes based on their name rather than the traditional node IDs, such as IP addresses. MVR stores hash information at backbone nodes to promote the routing transmission performance. In addition, the content routing mechanism also attracts attentions in traditional Internet [7], routers buffer information for some contents, and the service requests are embedded into the Internet name system. Therefore, routers can provide services for the content requests, which greatly enhance network transmission efficiency. The contentbased routing techniques need relatively large changes in network equipment, and these changes are too difficult to be implemented in Internet. However, WSNs usually construct their specialized network systems in local regions, which can provide convenience for the content-based transmission techniques.

Data transmission in WSNs can be divided into two types: upward transmission and downward transmission [2]. Current researches mainly focus on the upward flow, which is usually used to collect sensing data. Moreover, current works of content-based transmission optimization is mainly about upward data flow. In the actual deployment, downward transmission is also an important part of WSNs [8], which is often associated with sensing content. However, downward transmission is implemented mainly based on node IDs. The work of this paper is to study the performance optimization of downward transmission combined with sensing content.

In WSNs, the aggregation of sensing contents can lessen the communication traffic and shorten the total length of transmission paths, which have been widely used in our work and life $[9,10]$. Gulluccio L et al. [11] proposed a data aggregation mechanism with adaptability during transmission, which deploys aggregation nodes based on congestion prediction and supports their dynamic moves. Ngai E et al. [12] presented a reliable collection mechanism for sensing data in wireless sensor-actuator networks, which considers transmission reliability from multiple factors including accuracy, importance, and data freshness. Wang C et al. [13] studied the capacity of data aggregation in WSNs and proposed an aggregation scheme in the tree-based routing system. In addition, data aggregation and content-based notification have been paid more attention in Pocket switched networks $[14,15]$.

Huang SC et al. [16] focused on the latency of data aggregation in WSNs and designed an algorithm according to the maximal independent sets, which achieves much less latency. In [17], Bagga $\mathrm{M}$ et al. proposed some approaches for semistructured and unstructured topologies to minimize the time latency of sensing data aggregation. In order to maximize the revenue of data gathering with a deadline in WSNs, Hariharan S et al. [18] proposed a corresponding algorithm with polynomial complexity. Kuo $\mathrm{T}$ W et al. [19] studied the construction of the data aggregation tree with the minimum energy cost and investigated two scenarios with and without relay nodes. Sometimes we use random routing for source privacy protection [20], although this will increase the transmission delay.

\section{Transmission Mechanism}

In Figure 1, the sensing content value of each node is given below the circle. If the sink node wants to send messages to some nodes whose sensing contents belong to $\langle 11,15\rangle$, how to do it? Apparently, the nodes who should process the message are $\mathrm{N}_{3}, \mathrm{~N}_{6}, \mathrm{~N}_{7}$. When only based on node identity (ID), the sink nodes have to send the message with the content range $<11,15>$ to all nodes. And then, every node decides whether or not to process it according to its own sensing value. If each root node stores all sensing contents of its subtree, the message transmission could be fulfilled easily. However, this 


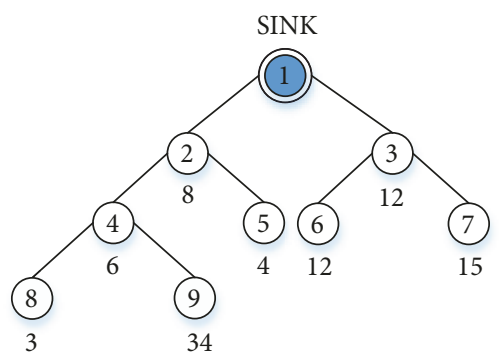

FIGURE 1: An example topology containing node sensing values.

storage mechanism would cost too much memory and have poor scalability. For instance, in Figure $1, \mathrm{~N}_{2}$ store 5 sensing values of their subtree: $8,6,4,3$, and 34 . Thus, a decimal value range (DEVR) rather than lots of specific values could be designed to describe the sensing contents of a subtree, which is similar to the idea of content aggregation researches $[9,17,18]$. For example, $N_{2}$ store the range: $\langle 3,34>$, and $\mathrm{N}_{3}$ store the range: $\langle 12,15\rangle$. DEVR optimizes the storage performance, but the sensing range description for a subtree is not accurate enough. In this paper, we design a novel storage method for the sensing value to optimize the message transmission.

For the content-based multicast messages, DEVR might result in invalid transmission. In Figure 1, the value range of the subtree rooted as $\mathrm{N}_{2}$ is $\langle 3,34\rangle$, and $\mathrm{N}_{2}$ is the same. If the sink node wants to notify nodes whose sensing contents belong to $\langle 11,15\rangle$, the message will be sent to $\mathrm{N}_{2}$ and then be sent to $\mathrm{N}_{4}$. These invalid transmissions consume processing and bandwidth resources

For anycast messages, it needs to be sent to any node meeting requirement. DEVR would also lead to redundant bandwidth consumption. Because there is no way to know which path to reach nodes satisfying the content condition, messages are always sent to all possible subtrees. In Figure 1, the anycast message whose sensing content belongs to $<11$, $15>$ must be sent to $\mathrm{N}_{2}$ and $\mathrm{N}_{3}$. However, there is no node meeting the requirements in the subtree rooted as $\mathrm{N}_{2}$. This is the problem that this paper needs to solve.

\subsection{Symbols Definition}

Definition 1. Continuous sensing value (C_Value) is used to describe a quantitative sensing data. For instance, the PM2.5 in the air and ambient noises both belong to C_Value. Ordinarily, the former is about in $\langle 0,1000\rangle$, and the latter is in $<0,200\rangle$.

Definition 2. Discrete sensing value (D_Value) is used to describe a sensing status about environment, equipment, human body, etc., which is one of several explicit status values. For example, human activity state (static, walking, and running) and working state of electronic equipment (power on, power off, and standby) both belong to D_Value.

In this paper, we design a sensing content storage method with " $0 / 1$ " bits called BIM, which applies for C_Value and D_Value at the same time.
For D_Value, $n$ bits represent the values of $n$ discrete sensing contents. The bit values of D_Value may be mutually exclusive or not. In the former case, there is only one "1". While in the latter case, there may be multiple "1".

For C_Value, each bit represents one value range. It is assumed that the value range of sensing contents is < $v_{\text {low }}, v_{\text {high }}>, v_{\text {low }} \leq v_{\text {high }}$. According to the precision requirement of the WSN environment, we define the value range represented by one bit: len. Then, the number of bits required in the environment is defined as $n_{b i t}$ and satisfies formula (1).

$$
n_{b i t}=\left\lceil\frac{\left(v_{\text {high }}-v_{\text {low }}+1\right)}{\text { len }}\right\rceil
$$

Assuming the value of a sensing content is $V_{k}, V_{\text {low }} \leq V_{k}<$ $V_{\text {high }}$, then its bit position is defined as $P O S_{b i t}$. We set " 1 " at the corresponding bit of C_Value: $P O S_{b i t}$. Apparently, $P O S_{b i t}$ can be calculated according to $V_{k}$, namely,

$$
\operatorname{POS}_{b i t}=\left\lceil\frac{\left(v_{k}-v_{l o w}+1\right)}{l e n}\right\rceil
$$

For instance, some sensors are used to monitor the environment temperature, and its value range for sensing value is $<1$, $40>$. As shown in Figure 2, we use each bit to represent five temperature values. According to formula (1), $n_{b i t}=\lceil(40-$ $1+1) / 5\rceil=8$, so 8 bits are needed to store the sensing content for the above value range. Then, in light of formula (2), the corresponding $P O S_{b i t}$ of the sensing content $" ~ 3{ }^{\circ} \mathrm{C}$ " is $1,\left(P O S_{b i t}=\lceil(3-1+1) / 5\rceil=1\right)$, and the $P O S_{b i t}$ of " $34^{\circ} \mathrm{C}^{\prime}$ " is 7 , $\left(P O S_{b i t}=\lceil(34-1+1) / 5\rceil=7\right)$. Therefore, " $3{ }^{\circ} \mathrm{C}$ " and " $34^{\circ} \mathrm{C}$ " are represented as "10000000" and "00000010", respectively.

In this paper, BIM uses each bit to represent a value range. Compared with traditional DEVR, BIM improves the description accuracy of sensing contents under certain conditions. According to the example shown in Figure 2, it is assumed that there are two sensor nodes in a subtree whose temperatures are $3^{\circ} \mathrm{C}$ and $34^{\circ} \mathrm{C}$. In DEVR, the merging value is expressed as $\langle 3,34>$, and it is "1000010" in BIM.

Definition 3. Content range (CR) depicts a specific sensing content, which includes a number of " $0 / 1$ " bits.

$C R$ is used in the following 2 cases:

(1) When stored in a node, $C R$ describes the sensing content range of one sensor node or a subtree with many nodes. For any tree node $N_{k}$, it stores $C R$ of each direct son node. Then, $N_{k}$ calculates and stores the $C R$ of the subtree rooted at itself.

(2) When embedded in notification messages, $C R$ describes the rule of the processing nodes. A contentbased notification message always carries a $C R$ that is used to restrict the sensor nodes dealing with it.

Definition 4. Multicast Message Based on Sensor Content $\left(M S G_{m u l t i}\right)$ is a kind of notification message sent to a set of sensor nodes whose sensing value matches the $C R$ carried by 


\begin{tabular}{|c|c|c|c|c|c|c|c|}
\multicolumn{1}{c}{ 1st } & \multicolumn{1}{c}{ 2nd } & \multicolumn{1}{c}{3 3rd } & \multicolumn{1}{c}{4 th } & 5th & 6th & 7th & 8th \\
\hline $1 \sim 5$ & $6 \sim 10$ & $11 \sim 15$ & $16 \sim 20$ & $21 \sim 25$ & $26 \sim 30$ & $31 \sim 35$ & $36 \sim 40$ \\
\hline
\end{tabular}

Figure 2: A storage example of BIM.

the message. $M S G_{m u l t i}$ is a broadcasting message when its $C R$ covers the whole content value range, which is received and processed by each node.

Definition 5. Anycast Message Based on Sensor Content $\left(M S G_{a n y}\right)$ is a kind of notification message sent to any one of the sensor nodes whose sensing value matches the $C R$ carried by the message. $M S G_{a n y}$ only need to be processed by one node.

Note that all notification messages are regarded to be sent from sink node.

\subsection{Transmission Mechanism Design}

3.2.1. Contents Aggregation. In CEMT, it must be first implemented that sensing contents are collected and aggregated at roots of all subtrees in WSNs, which forms the base of content-based message transmission. In tree-like topologies, nodes implement sensing contents aggregation periodically in any subtree. Each node, on behalf of all nodes, belongs to its subtree and reports the $C R$ of the whole subtree to the direct father node at regular intervals. Therefore, any node in the tree topology stores the $C R$ of the subtree rooted as it. Set the reporting period for the sensing contents as $T_{\text {report }}$, and the node roles are defined as follows.

(1) Leaf node reports the latest sensing value to the direct father node at each period of $T_{\text {report }}$.

(2) Sink node monitors the sensing contents reported by direct son nodes and then renews and stores $C R$ of each subtree.

(3) Other nodes (neither leaf node nor sink node). On the one hand, the node monitors the sensing contents reported by direct son nodes and then renews and stores CRs of each subtree. On the other hand, it aggregates $C R s$ of all son nodes and itself before reporting the aggregated $C R$ of the subtree rooted as it to its direct father node at each period of $T_{\text {report }}$.

\subsubsection{Messages Transmission Based on Sensing Contents.} Generally, the message transmission in WSNs is grouped into two types in terms of transmission direction: (1) being sent to sensor nodes from sink node; (2) being sent to sink node from sensor nodes. In the tree topology of this paper, the above two types of transmissions are upstream messages from tree nodes to root node and downstream messages from root node to leaf nodes (destination may be a nonleaf node). Upstream messages are always sent along parent nodes, which is unassociated with sensing content and relatively simple. Therefore, we omit the discussion of upstream messages and only focus on downstream multicast messages and anycast ones.

We take Figure 3 as an example to introduce the transmission procedure of content-based messages. Each node has sensing content value of itself, which is labeled under the node (ellipse). In addition, each node stores the aggregated $C R$ of its subtree, which is labeled above the node (rectangle).

(1) Transmission of $M S G_{\text {multi }}$. The message transmission starts from sink node, which sends the message to all subtrees satisfying content conditions in view of CRs of each subtree stored in them. After that, the above work is implemented to tree leaf nodes layer by layer. For example, in Figure 3, sink node $\left(\mathrm{N}_{1}\right)$ sends a $M S G_{\text {multi }}(C R=10000000)$ to all nodes satisfying the requirement. Blue blocks describe the sending procedure in Figure 3. Note that since $\mathrm{N}_{3}$ does not meet the $C R$ condition, it only forwards the message without dealing with it.

(2) Transmission of $M S G_{a n y}$. A $M S G_{a n y}$ starts from the sink node too. It is sent to a certain subtree satisfying content requirement. The transmission is implemented to tree leaf layer by layer. When a node receives a $M S G_{a n y}$, it will stop the transmission if the sensing content $(C R)$ of itself meets the rule of the $M S G_{a n y}$. For instance, in Figure 3, the sink node $\left(\mathrm{N}_{1}\right)$ sends the $M S G_{\text {an } y}(C R=01000000)$ in the tree topology. Red blocks describe the sending procedure in Figure 3. Note that the transmission procedure is stopped in $\mathrm{N}_{9}$ because $\mathrm{N}_{9}$ satisfies the $C R$ carried by the $M S G_{a n y}$. Moreover, because the $M S G_{a n y}$ only needs to be sent to one receiving node, N4 send it to $\mathrm{N}_{9}$ and do not send it to $\mathrm{N}_{10}$.

\section{Detailed Design}

In CEMT, IP unicast rather than IP multicast is used to transmit IP packets between adjacent nodes. The main considerations are as follows. At first, messages transmission may correspond to different IP multicast groups so that the protocol maintenance cost for multicast groups is high. Moreover, IP multicast transmission brings additional work on unrelated nodes which spend lots of resources on invalid packets processing.

4.1. Sensing Contents Aggregation. For a nonleaf node, $N_{k}$, it receives sensing contents messages reported by its son nodes. Firstly, it records $C R$ of each son node, and this information constitutes content routing entries (RTS) for subtrees, which is expressed as

$$
R T S_{i}=\left\langle C R_{i}, I P_{i}\right\rangle, \quad 1 \leq i \leq n .
$$




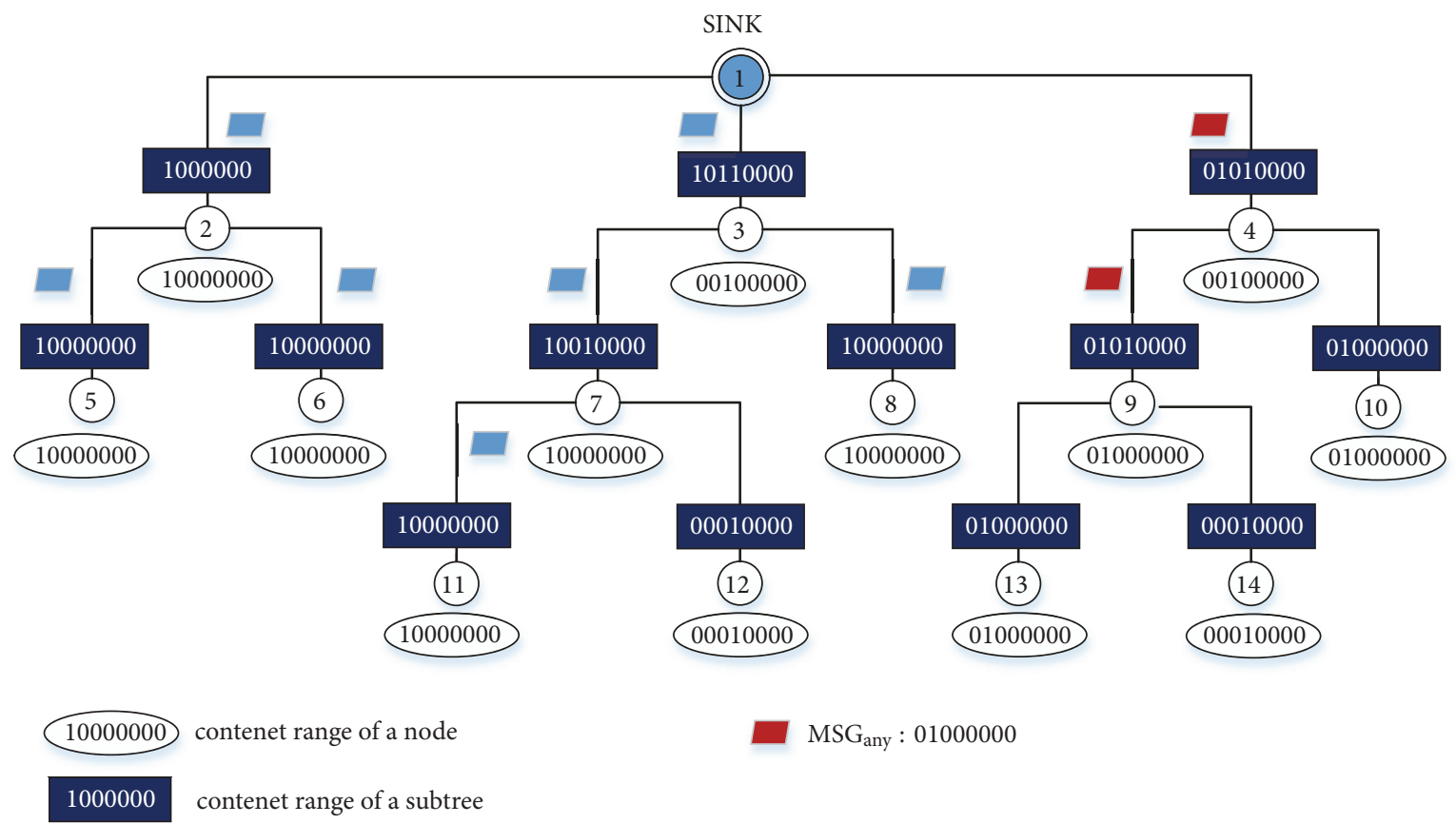

MSG $_{\text {multi }}: 10000000$

FIGURE 3: The transmission of the $M S G_{m u l t i}$ and $M S G_{a n y}$ in CEMT.

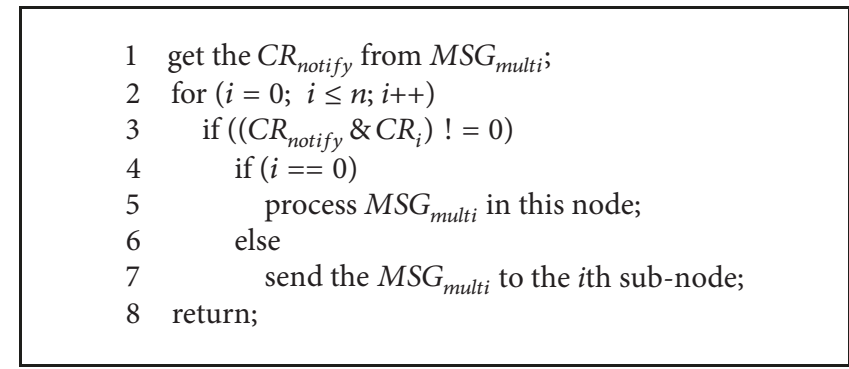

Algorithm 1: Multicast_notification_deal $\left(M S G_{m u l t i}\right)$.

Among them, $n$ is the number of son nodes of $N_{k}$, and $R T S_{i}$ is the $i$ th routing entry for its $i$ th subtree.

At the same time, $N_{k}$ has $C R$ of itself, which is stored as $R T S$ for processing convenience, i.e., $R T S_{0}=\left\langle C R_{0}, I P_{0}\right\rangle$. Note that $I P_{0}$ has no practical significance, and the value of which is set to 0 .

After that, $N_{k}$ aggregates CRs of all its subtrees and itself, which is described as formula (4). “ $\oplus$ ” is an "OR" operation symbol for " $0 / 1$ ". Certainly, $N_{k}$ also reports the aggregated $C R$ to its parent node.

$$
C R_{\text {subtree }(k)}=C R_{0} \oplus C R_{1} \oplus C R_{2} \oplus \cdots \oplus C R_{n}
$$

In Figure 3, the $C R$ of $\mathrm{N}_{3}$ is " 00100000 ", and it has two subtrees whose CRs are "10010000" and "10000000". Therefore, the $C R_{\text {subtree (3) }}$ equals " 10110000 ", which is calculated as formula (5).

$$
C R_{\text {subtree }(3)}=00100000 \oplus 00100000 \oplus 10000000
$$

4.2. Processing of $M S G_{\text {multi }}$. When $N_{k}$ receives a $M S G_{\text {multi }}$, it will get the content range from the packet, that is, $C R_{\text {notify }}$. Firstly, $N_{k}$ judges whether it needs to process the message itself. If the result of $C R_{0}$ and $C R_{\text {notify }}$ is greater than 0 , it can be concluded that $N_{k}$ meets the content condition of the notifying message, and the message should be processed by $N_{k}$. Moreover, $N_{k}$ analyzes whether each subtree satisfies $C R_{\text {notify }}$ and forwards the $M S G_{\text {multi }}$ to all subtrees matching the content rule. The basic algorithm of processing the $M S G_{\text {multi }}$ is described in Algorithm 1.

4.3. Processing of $M S G_{a n y}$. The processing procedure is similar to that of the $M S G_{\text {multi }}$. When $N_{k}$ receives a $M S G_{a n y}$, it will get the content range from the packet, that is, $C R_{\text {notify }}$. At first, $N_{k}$ judges whether there is a need to process the message by itself. The judging method is the same as the above description. If $N_{k}$ matches the content rule of the $M S G_{a n y}$, it will process the notifying message without forwarding it to any other nodes. Otherwise, $N_{k}$ selects an arbitrary subtree 


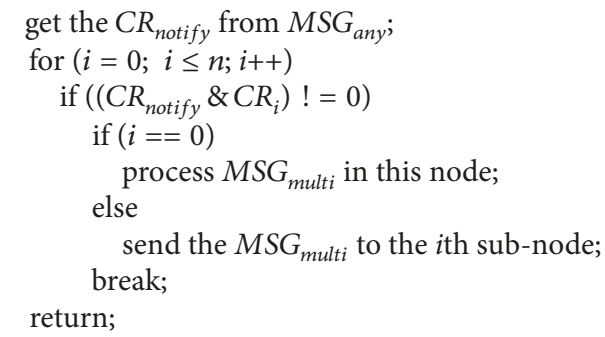

Algorithm 2: Anycast_notification_deal $\left(M S G_{a n y}\right)$.

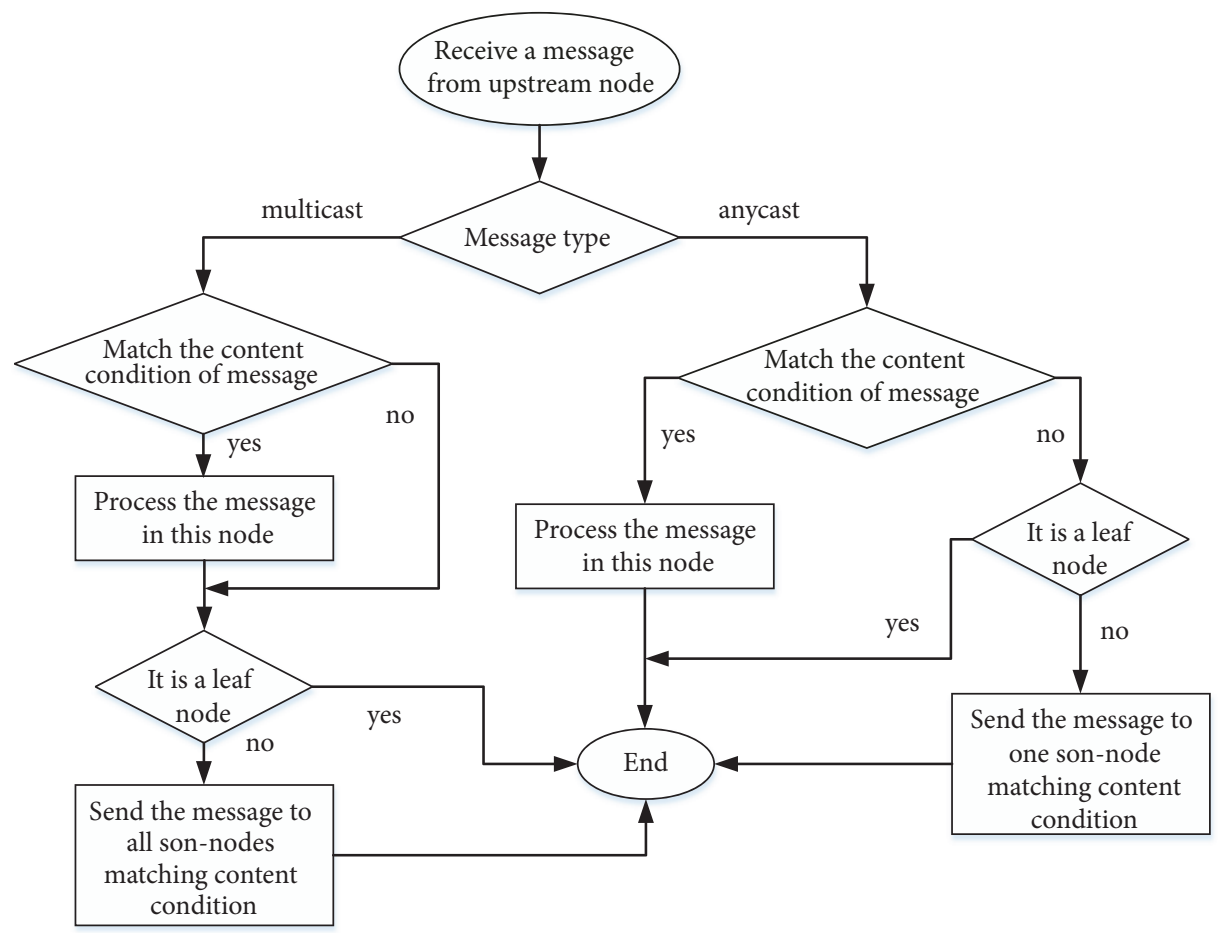

FIGURE 4: Message processing flow in the sensor node.

satisfying the content rule and forwards the message to the corresponding son node. Note that $N_{k}$ forwards the $M S G_{a n y}$ to only one subtree because it is an anycast message. The basic algorithm of processing the $M S G_{a n y}$ is described in Algorithm 2.

In summary, the message processing flow in CEMT is given as Figure 4.

\section{WSNs with Multidimensional Sensing Contents}

In WSNs, we often deploy various kinds of sensor nodes to monitor different sensing contents, which constitute a subnet together. These nodes provide packets forwarding services for each other, which greatly enhances utilization of network transmission resources in LLN (low-power and lossy networks). As shown in Figure 5, temperature and light are monitored at the same time in a WSN subnet. Circular nodes and square nodes monitor temperature and light of

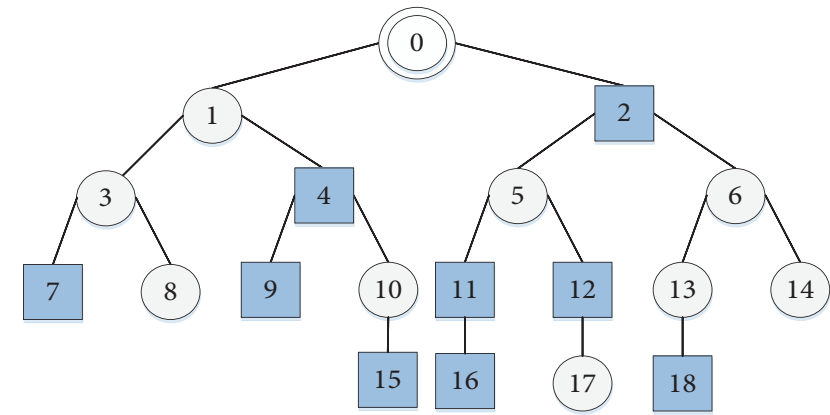

FIGURE 5: A WSN subnet with multiple types of sensor nodes.

the corresponding circumstances, respectively. Two types of nodes are mixed together to construct networks in order to reduce the number of sensor nodes and extend the sensing scope. Undoubtedly, the sink node (tree root) supports both sensing contents. 


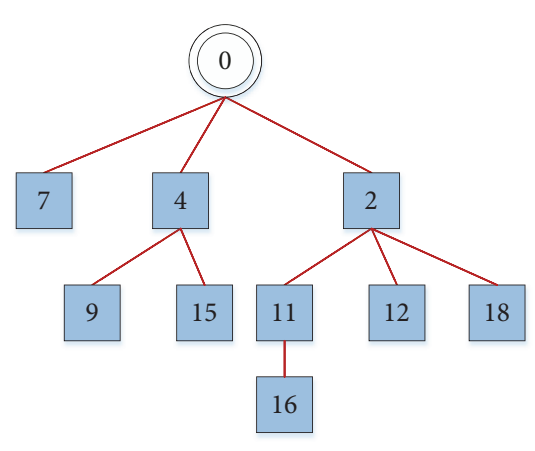

(a) Light

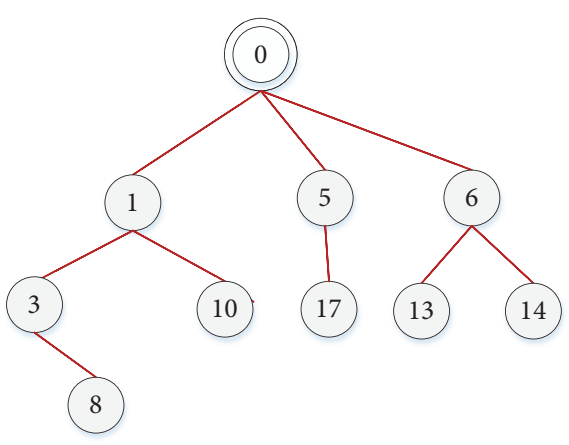

(b) Temperature

Figure 6: The logical trees with the same sensing content types.

While transmitting content-based messages, CEMT constructs a special logical tree for the same sensing contents, and notification messages are transmitted along the tree. Intrinsically, constructing the logical tree is to form the logical parent-child relationships among sensor nodes with the same sensing contents.

Definition 6. Belonging relationship of nodes: if $N_{i}$ is in the subtree rooted as $N_{j}, N_{i}$ belongs to $N_{j}$, or $N_{j}$ administer $N_{i}$, which is expressed as $N_{i} \prec N_{j}$. In Figure 5, some belonging relationships are denoted as $N_{3} \prec N_{1}, N_{9} \prec N_{1}$.

Definition 7. Logical father-son relationship: $N_{i}$ and $N_{j}$ are the same kind of nodes in tree topologies. $N_{i}$ is the son node of $N_{j}$ which is expressed as $N_{i} \triangleleft N_{j}$. Certainly, $N_{j}$ is the father node of $N_{i}$. then

Given two nodes of the same type: $N_{i}, N_{j}$, and $N_{i} \prec N_{j}$,

$$
\neg\left(\exists N_{k} \mid N_{i} \prec N_{k} \wedge N_{k} \prec N_{j}\right)=>N_{i} \triangleleft N_{j}
$$

The methods judging the logical father-son relationship are as follows.

(1) Logical father node of $N_{k}$ is the nearest node with the same type to $N_{k}$ on the path (only one path) from $N_{k}$ to the root node. "The nearest" means the minimum hops. Obviously, the root node of a tree has no father node, and a nonroot node has one and only one logical father node. In Figure 5, the father node of $\mathrm{N}_{11}$ is $\mathrm{N}_{2}$.

(2) Logical son node of $N_{k}$ is the nearest node with the same type on the path from $N_{k}$ to each leaf node. Obviously, in a logical tree, any nonleaf node has one or more logical son nodes. There may be a logical son node on each path to leaf nodes. In Figure 5, $\mathrm{N}_{1}$ has two son nodes: $\mathrm{N}_{3}$ and $\mathrm{N}_{10}$.

In light of the above analysis, the WSN subnet with multiple types of sensor nodes can be split into multiple logical trees with different content types. For instance, the WSN subnets in Figure 5 are split into two logical trees shown as Figure 6. The lines in Figure 6 represent the logical father-son relationships rather than the physical connection.
In WSN environments with various types of sensor nodes, the transmission of content-based notification messages is implemented in view of the above-mentioned logical trees. Algorithm 3 depicts how a node gets its logical son nodes. In this circumstance, one node may have so many logical son nodes that its load is too heavy. Certainly, we can adjust the tree topology to avoid this problem. This paper omits the details here.

Because IP unicast is used to transmit IP packets between a father node and its son nodes, content aggregation and message transmission are compatible with the mechanism described in Section 3. In Figure 5, it is assumed that $\mathrm{N}_{0}$ sends a $M S G_{\text {multi }}$ to $\mathrm{N}_{4}$ and then $\mathrm{N}_{4}$ forwards it to $\mathrm{N}_{9}$. Therefore, two unicast IP packets exist. The source IP and destination IP of the first two packet are $\left(\mathrm{IP}_{0}, \mathrm{IP}_{4}\right)$ and $\left(\mathrm{IP}_{4}, \mathrm{IP}_{9}\right)$, respectively.

\section{Experiments and Analyses}

Table 1 shows a list of notations used in the following analysis. Set the layer number of sink nodes to be 0 and take the layer number of the lowest node equal to $L_{\max }$. Consequently, there are total $\left(L_{b}+1\right)$ layers in the tree-like topology. CEMT introduces extra storage cost for RTSs (content routing entries for subtrees). IP addresses of direct son nodes are inherently stored in nonleaf nodes, which can be utilized for RTS. Therefore, the extra storage cost of each node in CEMT is mainly CRs (content range) of its direct subtrees. In general, one $C R$ only needs 1 byte. We analyze average storage cost of nodes on the same layer, which is shown in formula (7).

$$
A V S_{i}= \begin{cases}S *\left(\frac{N_{i+1}}{N_{i}}+1\right), & i<L_{b} \\ S, & i=L_{b}\end{cases}
$$

If the topology is a full $n$-ary tree, the storage cost of each node is described in formula (8). Each leaf node only stores one $C R$ for itself. Obviously, storage cost of one node in CEMT is only related to the number of its direct subtrees and does not change with the number of its descendants nodes.

$$
S_{i}= \begin{cases}S * n, & i<L_{b} \\ S, & i=L_{b}\end{cases}
$$




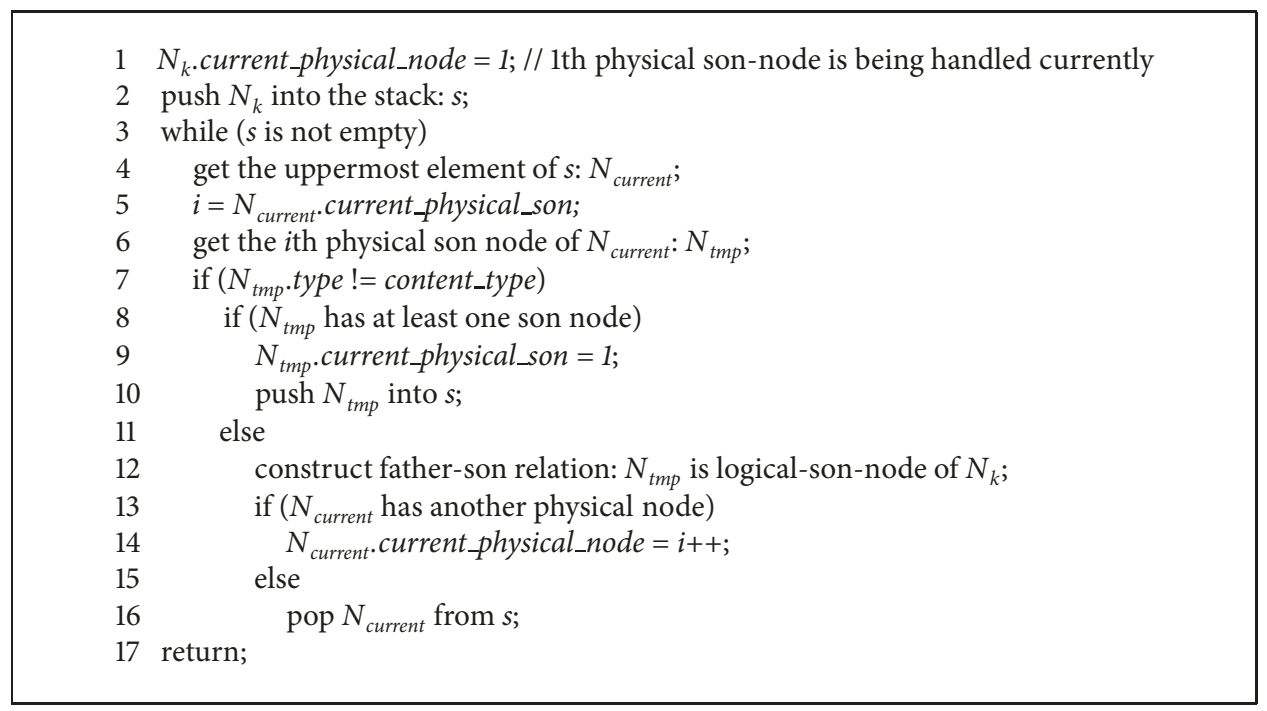

Algorithm 3: Get_logic_son_nodes $\left(N_{k}\right.$, content_type $)$.

TABLE 1: Notations for analysis.

\begin{tabular}{lc}
\hline Symbols & Description \\
\hline$L_{b}$ & layer number of nodes at the bottom of topology tree \\
$S$ & number of bytes of one "content range" \\
$N_{i}$ & nodes number in ith layer \\
$A V S_{i} / S_{i}$ & (average) storage for content range of one node in $i$ th layer \\
$S T_{j}(C R)$ & The number of subtrees in $j$ th layer satisfying $C R$ (average value) \\
$L_{\min }(C R) / L_{\max }(C R)$ & The layer number (average value) of the node which is the nearest /the farthest away from sink in every branch
\end{tabular}

We analyze the number of nodes dealing with one content message for a given $C R$. When $L_{\text {max }}(C R)=0$ or $L_{\text {min }}(C R)=$ 0 , no node satisfies the content condition. For a $M S G_{a n y}$, the number of nodes handling it is $L_{\text {min }}(C R)+1$. When $L_{\text {min }}(C R)=0$, only sink nodes deal with the $M S G_{\text {any }}$. For a $M S G_{m u l t}$, the number of nodes handling the message is

$$
1+\sum_{i=1}^{l_{\text {max }}(C R)} \overline{S T}_{i}(C R)
$$

The key contributions of CEMT are to lessen the number of processing nodes and reduce the transmission time of content-based messages. We carry out related experiments and analyze through MATLAB. Topologies of complete $n$ ary trees are used to analyze the performance of CEMT. Sensing content values of nodes are generated randomly. We compare three transmission methods: CEMT, DEVR, and the traditional routing mechanism based on node identity (TRID). For the same topology and sensing contents, we analyze and compare the results of three methods, including processing node number and the transmission delay of notification messages.

When a node sends a message to its multiple descendant nodes, we assume that it carries out transmissions from left to right, and all nodes receiving the message deal with it in parallel. At the same time, the time that the node sends to the child node is always equal: 1 unit time. In Figure $7, \mathrm{~N}_{1}$ wants to advertise a message to all nodes. Then, how many units of time each node gets the message are marked above the node. For instance, $\mathrm{N}_{5}$ gets the message in the $3 \mathrm{rd}$ unit time. For instance, $\mathrm{N}_{5}$ gets the message in the 3 rd cycles from the beginning of transmission at $\mathrm{N}_{1}$.

Aiming at the above performance indicators, three mechanisms including CEMT, DEVR, and TRID are compared. In Figure 8, we analyze the number of nodes processing messages and transmission delay through a network with 50 sensor nodes. In these experiments, the nodes are constructed into a 3 -ary tree, and all sensing values are generated randomly.

In Figure 8(a) for transmission of the $M S G_{\text {multi }}$, TRID must send the $M S G_{\text {multi }}$ to all nodes to process it. In contrast, CEMT and DEVR only need a few nodes to process the message based on content range rules, and the former is fewer because of its more accurate description of the sensing contents. According to our measurements, the average transmission delay of a single hop is about $0.05 \mathrm{~s}$. Thus, the whole transmission delay of one message could be calculated. In Figure 8(b), CEMT still has the best performance on transmission delay for the $M S G_{m u l t i}$, and it usually takes less time than half of TRID.

The experiments results for $M S G_{\text {any }}$ are shown in Figures $8(\mathrm{c})$ and $8(\mathrm{~d})$. In most cases, CEMT makes the message processing nodes fewer while ensuring the completion of notification transmission. In general, its transmission delay is also less than other two mechanisms.

As we can see, there are some overlapping points between CEMT and DEVR in Figures 8(a) and 8(b). For the two 


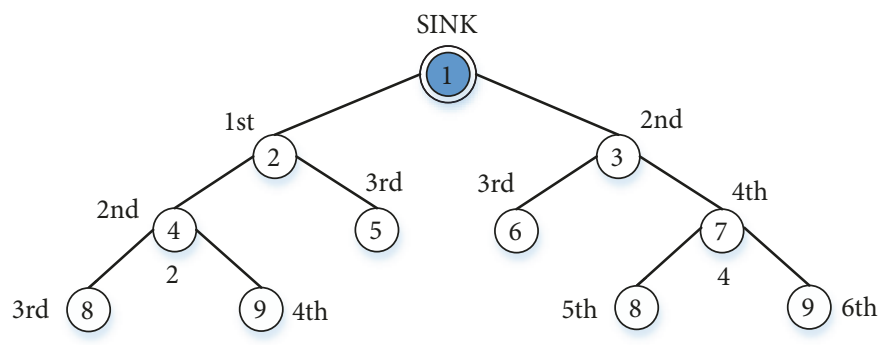

FIgURE 7: An example for transmission cycles.
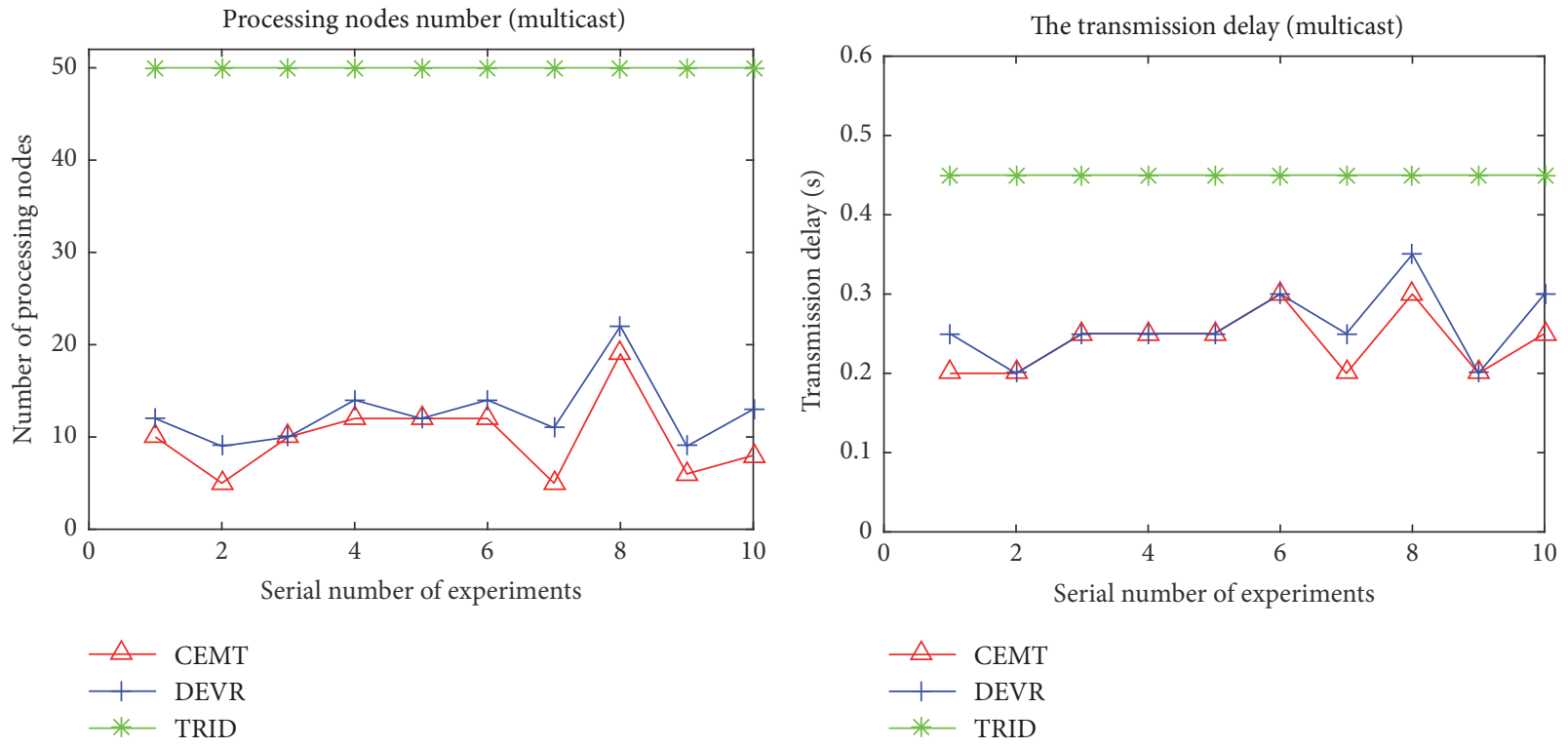

(a)
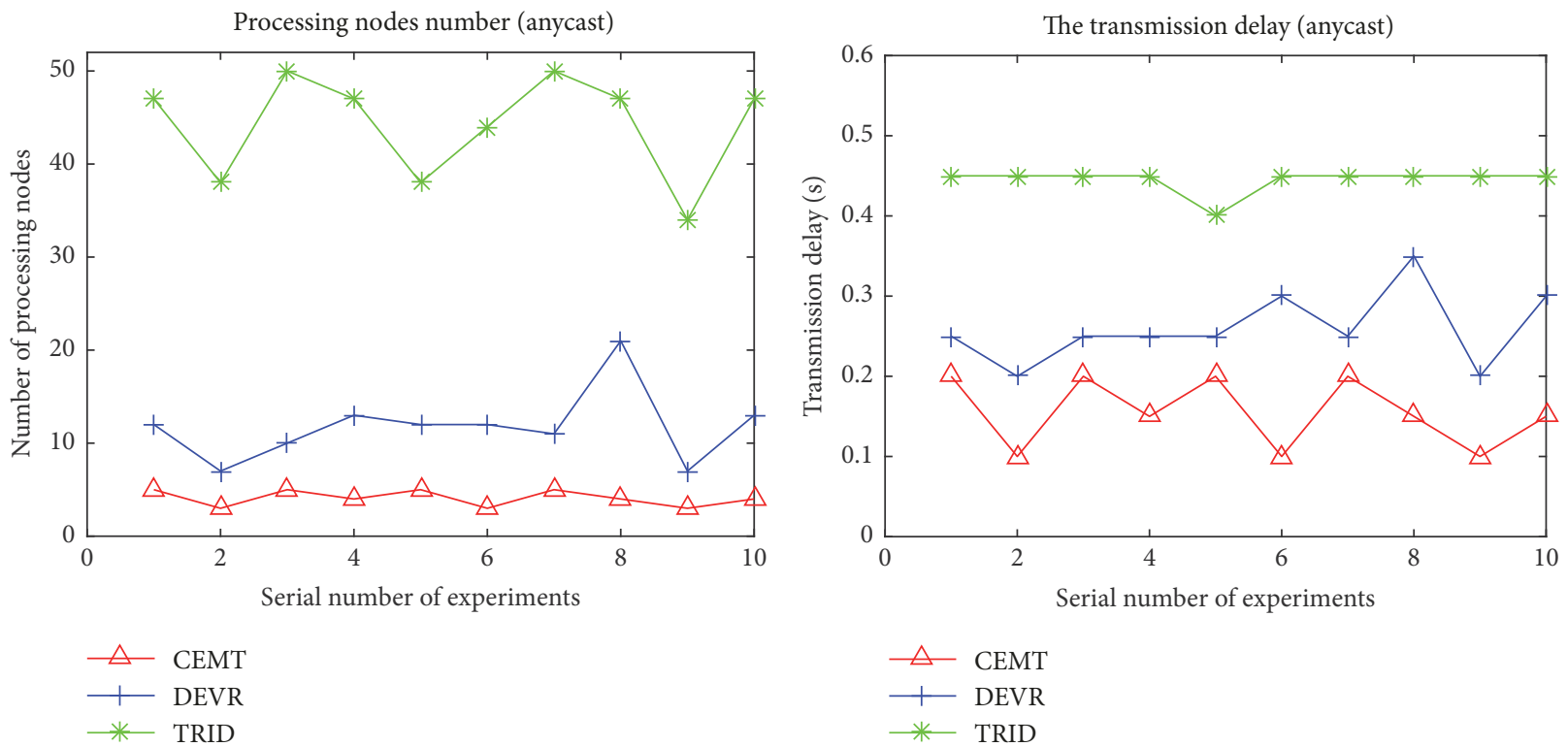

(c)

(d)

FIGURE 8: Processing nodes number and transmission delay in 3-ary tree. 


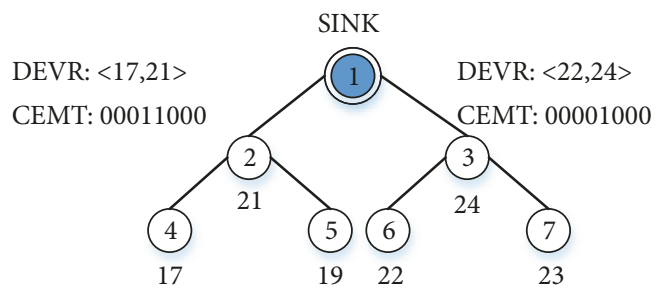

(a)

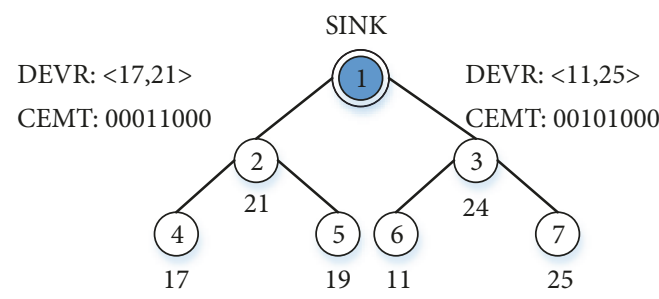

(b)

FIGURE 9: Analysis of processing nodes for two mechanisms.
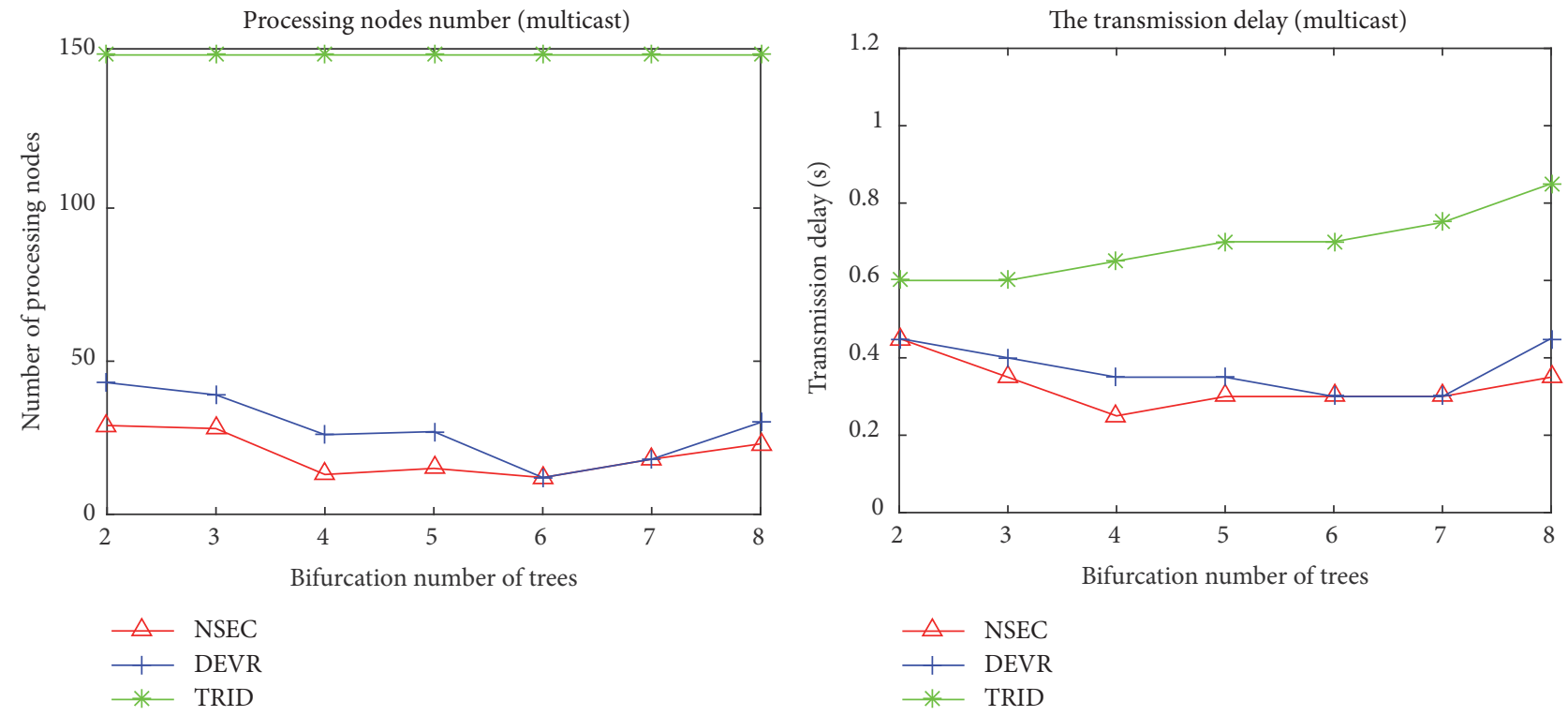

(a)

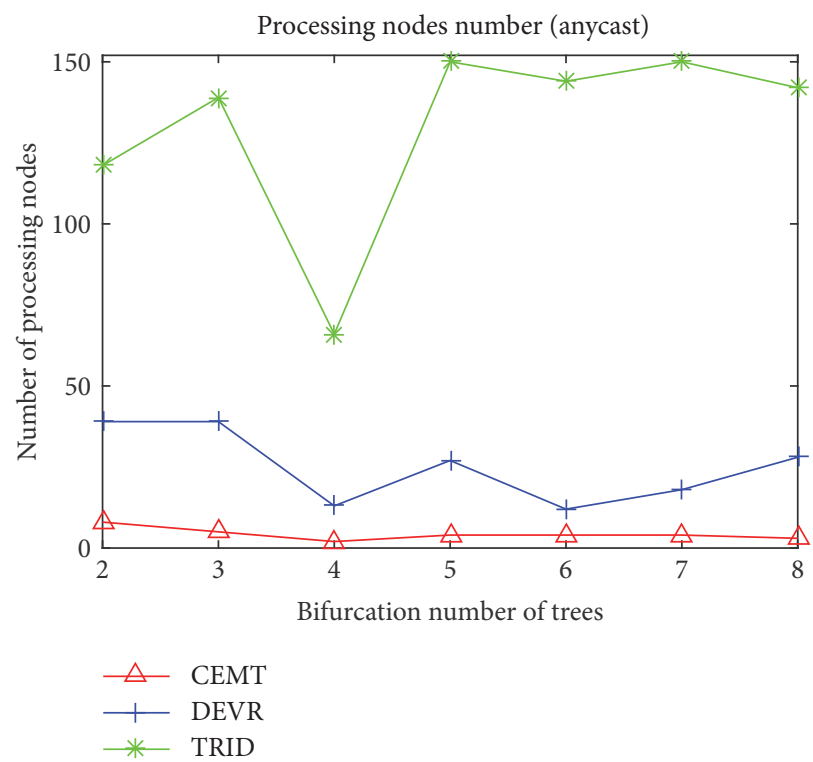

(c)

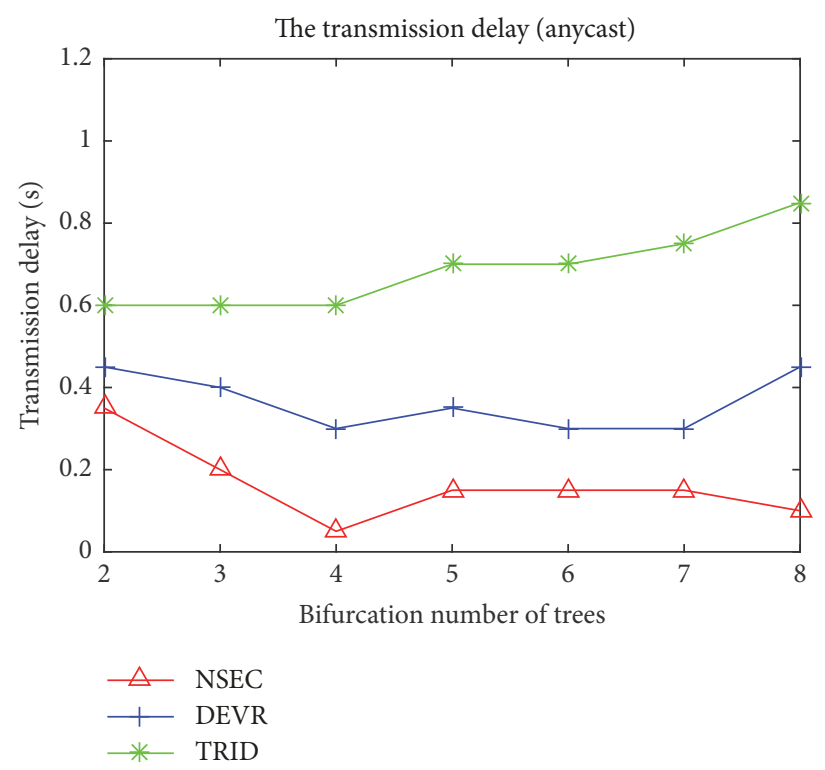

(d)

FIGURE 10: Processing nodes number and transmission delay in trees with different bifurcation number. 
mechanisms, the number of nodes processing a message is related to 3 factors: the network topology, sensing contents of nodes, and the $C R$ of the message. In most cases, there are fewer processing nodes for a message in CEMT than in DEVR. However, sometimes there are the same processing nodes in two mechanisms.

In the example shown in Figure 9, the range of the temperature sensing value is $\langle 1,40\rangle$. In CEMT, each bit represents 5 temperature units. In addition, the CRs stored in the key nodes $\left(\mathrm{N}_{2}, \mathrm{~N}_{3}\right)$ have been given in the figures. The sink node wants to send nodes a $M S G_{\text {multi }}$. The $C R$ of the message is $\langle 16,19\rangle$ in DEVR, which is equivalent to “00010000" in CEMT. In Figure 9(a), no matter in CEMT or DEVR, there are 3 nodes processing the $M S G_{\text {multi }}: \mathrm{N}_{2}, \mathrm{~N}_{4}$, and $\mathrm{N}_{5}$. However, in Figure 9(b), the $M S G_{\text {multi }}$ will be sent to 3 nodes, $\mathrm{N}_{2}, \mathrm{~N}_{4}$, and $\mathrm{N}_{5}$, in CEMT, and sent to 4 nodes, $\mathrm{N}_{2}$, $\mathrm{N}_{3}, \mathrm{~N}_{4}$, and $\mathrm{N}_{5}$, in DEVR.

Then, we analyze processing nodes number and transmission delay for one notification message in trees with different bifurcation number (3-ary tree $\sim 8$-ary tree). As shown in Figure 10, CEMT always achieves the best performance among three mechanisms.

\section{Conclusions}

Transmission of notification messages is implemented mainly around sensing contents. In this paper, we present a notification transmission mechanism based on sensing content (CEMT) in WSNs to improve the notifying efficiency, such as reducing processing nodes, transmission time, and energy consumption. At first, sensing contents are divided into two types: continuous sensing value and discrete sensing value. After that, a sensing content storage method called BIM is proposed, which can support the above two values simultaneously. In addition, we define two kinds of notification messages: multicast messages and anycast messages. CEMT collects and aggregates sensing contents at root nodes of all subtrees. Thus, CEMT accurately sends messages to nodes meeting corresponding rules through content routing of tree nodes. Furthermore, in CEMT, various kinds of sensor nodes monitoring different sensing contents may be mixed to make up a subnetwork. In this environment, we construct multiple logical trees with the same sensing contents, and notification messages are transmitted along their logical trees.

\section{Data Availability}

Our experiments data could be obtained through URL: https://pan.baidu.com/s/1obHn_cak2jMkr6YLHCOlrw, PWD: h5eu.

\section{Conflicts of Interest}

The authors declare that they have no conflicts of interest.

\section{Acknowledgments}

This research is supported by the National Natural Science Foundation of China (61373161 and 61272446) and Beijing Natural Science Foundation (4172019).

\section{References}

[1] Routing Over Low power and Lossy networks (ROLL). http://datatracker.ietf.org/wg/roll/documents.

[2] T. Winter, P. Thubert, A. Brandt et al., "RPL: IPv6 Routing Protocol for Low-Power and Lossy Networks," RFC Editor RFC6550, 2012.

[3] X. Rong, S. Bo, X. Yong kang, and W. Yungang, "A Notification Transmission Mechanism for Sensor Nodes Based on Sensing Contents in IoTs," in Proceedings of the International Conference on Identification, Information and Knowledge in the Internet of Things (IIKI), pp. 1-5, IEEE, Qufu, China, 2017.

[4] J. J. Garcia-Luna-Aceves, Q. Li, and T. Karadeniz, "CORD: Content oriented routing with directories," in Proceedings of the 2015 International Conference on Computing, Networking and Communications, ICNC 2015, pp. 785-790, USA, February 2015.

[5] J. J. Garcia-Luna-Aceves and Q. Li, "Efficient content routing in MANETs using distances to directories," in Proceedings of the 2014 IEEE Conference on Computer Communications Workshops, INFOCOM WKSHPS 2014, pp. 422-427, Canada, May 2014.

[6] M. Li and L. Gao, "Multi-level virtual ring: An architecture for content routing in wireless sensor network," in Proceedings of the 17th Asia Pacific Conference on Communications, APCC 2011, pp. 553-558, Malaysia, October 2011.

[7] Z. Ming, M. Xu, C. Xia, D. Li, and D. Wang, "SIONA: A service and information oriented network architecture," in Proceedings of the 2012 IEEE International Conference on Communications, ICC 2012, pp. 2650-2654, Canada, June 2012.

[8] A. Khalifeh, S. Al-Agtash, R. Tanash, and M. Alqudah, "Deploying agents for monitoring and notification of wireless sensor networks," in Proceedings of the 28th IEEE International Conference on Tools with Artificial Intelligence, ICTAI 2016, pp. 754757, USA, November 2016.

[9] S. Bose, N. Mukherjee, and S. Mistry, "Environment monitoring in smart cities using virtual sensors," in Proceedings of the 4th IEEE International Conference on Future Internet of Things and Cloud, FiCloud 2016, pp. 399-404, Austria, August 2016.

[10] R. J. Nayaka and R. C. Biradar, "Data aggregation and routing scheme for smart city public utility services using WSN," in Proceedings of the 2nd IEEE International Conference on Electrical, Computer and Communication Technologies, ICECCT 2017, India, February 2017.

[11] L. Galluccio, A. T. Campbell, and S. Palazzo, "Concert: aggregation-based congestion control for sensor networks," in Proceedings of the 3rd International Conference on Embedded Networked Sensor Systems, pp. 274-275, San Diego, California, USA, November 2005.

[12] E. H. Ngai, Y. Zhou, M. Lyu, and J. Liu, "Reliable Reporting of Delay-Sensitive Events in Wireless Sensor-Actuator Networks," in Proceedings of the 2006 IEEE International Conference on Mobile Ad Hoc and Sensor Sysetems, pp. 101-108, Vancouver, BC, Canada, October 2006.

[13] C. Wang, C. Jiang, Y. Liu, X.-Y. Li, and S. Tang, "Aggregation capacity of wireless sensor networks: extended network case," Institute of Electrical and Electronics Engineers. Transactions on Computers, vol. 63, no. 6, pp. 1351-1364, 2014.

[14] S. Wang, X. Wang, J. Huang, R. Bie, and X. Cheng, "Analyzing the potential of mobile opportunistic networks for big data applications," IEEE Network, vol. 29, no. 5, pp. 57-63, 2015. 
[15] S. Wang, M. Liu, X. Cheng, and M. Song, "Routing in pocket switched networks," IEEE Wireless Communications Magazine, vol. 19, no. 1, pp. 67-73, 2012.

[16] S. C.-H. Huang, P.-J. Wan, C. T. Vu, Y. Li, and F. Yao, "Nearly constant approximation for data aggregation scheduling in wireless sensor networks," in Proceedings of the 26th IEEE International Conference on Computer Communications (IEEE INFOCOM '07), pp. 366-372, IEEE, May 2007.

[17] M. Bagaa, A. Derhab, N. Lasla, A. Ouadjaout, and N. Badache, "Semi-structured and unstructured data aggregation scheduling in wireless sensor networks," in Proceedings of the IEEE Conference on Computer Communications, INFOCOM 2012, pp. 2671-2675, USA, March 2012.

[18] S. Hariharan and N. B. Shroff, "Maximizing aggregated revenue in sensor networks under deadline constraints," in Proceedings of the 48th IEEE Conference on Decision and Control, pp. 48464851, 2009.

[19] T.-W. Kuo, K. C. Lin, and M.-J. Tsai, "On the construction of data aggregation tree with minimum energy cost in wireless sensor networks: NP-completeness and approximation algorithms," Institute of Electrical and Electronics Engineers. Transactions on Computers, vol. 65, no. 10, pp. 3109-3121, 2016.

[20] W. Chen, M. Zhang, G. Hu, X. Tang, and A. K. Sangaiah, "Constrained Random Routing Mechanism for Source Privacy Protection in WSNs," IEEE Access, vol. 5, pp. 23171-23181, 2017. 


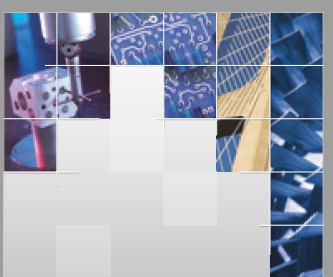

\section{Enfincering}
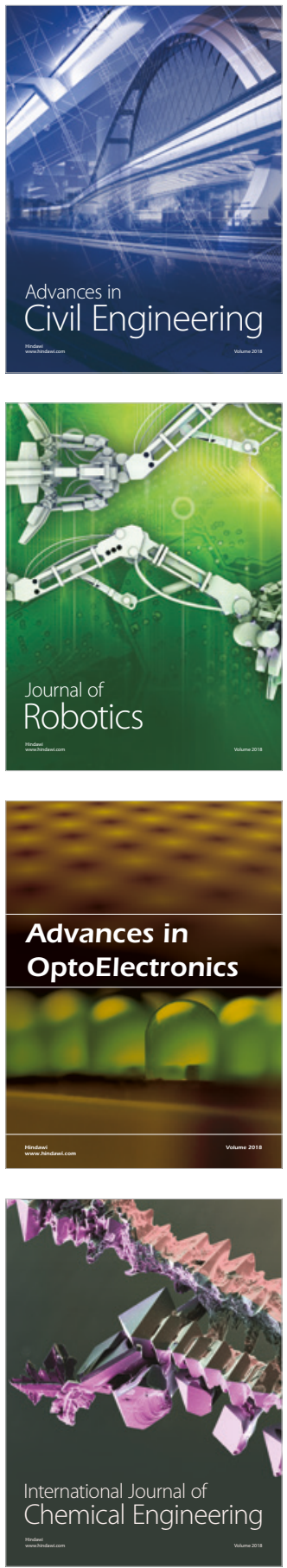

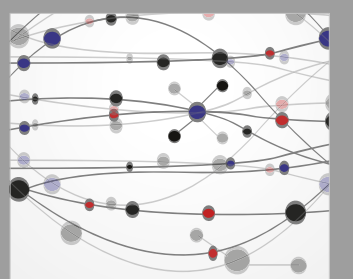

\section{Rotating \\ Machinery}

The Scientific World Journal

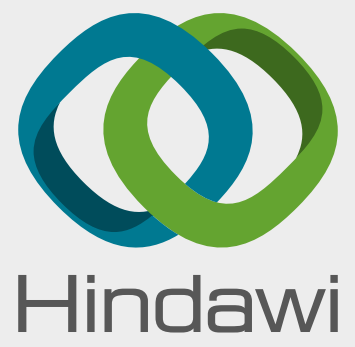

Submit your manuscripts at

www.hindawi.com
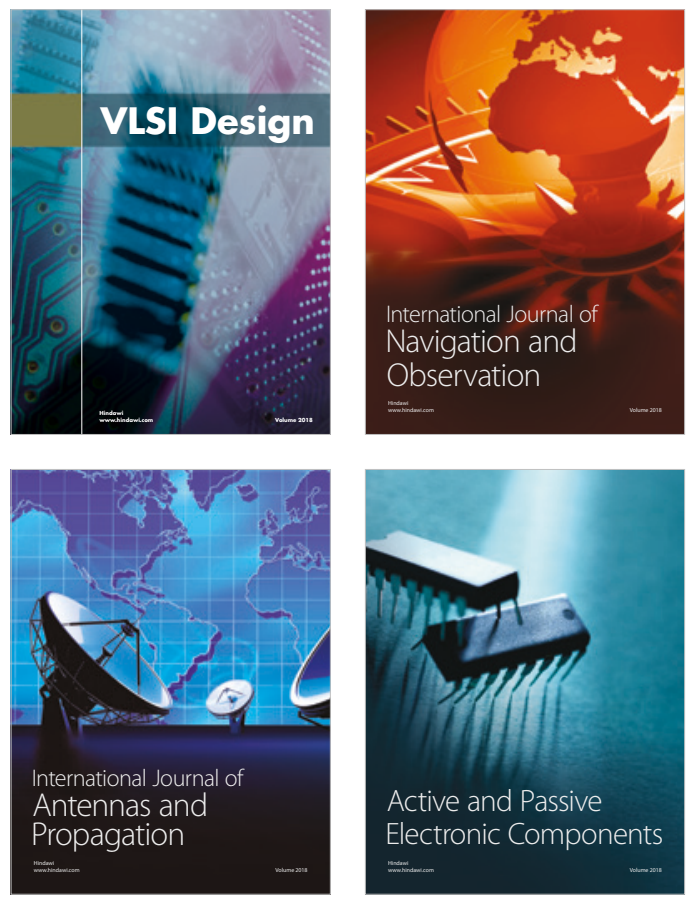
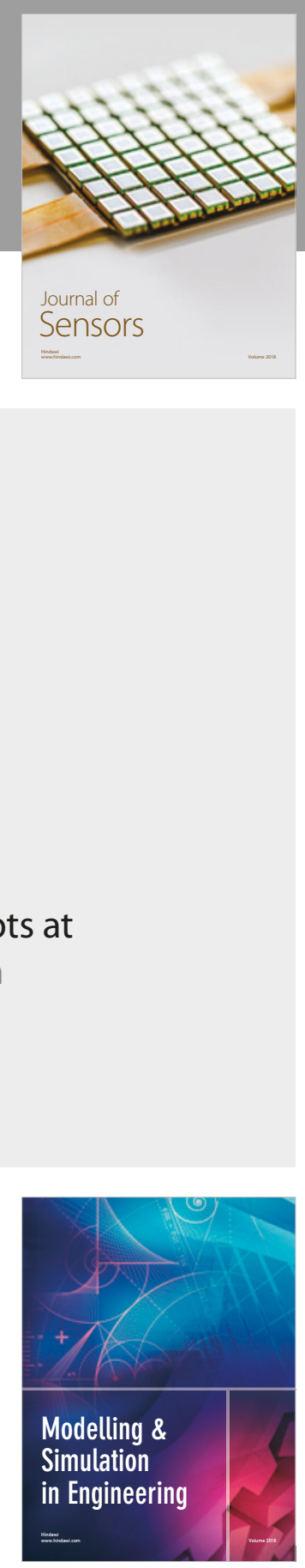

\section{Advances \\ Multimedia}
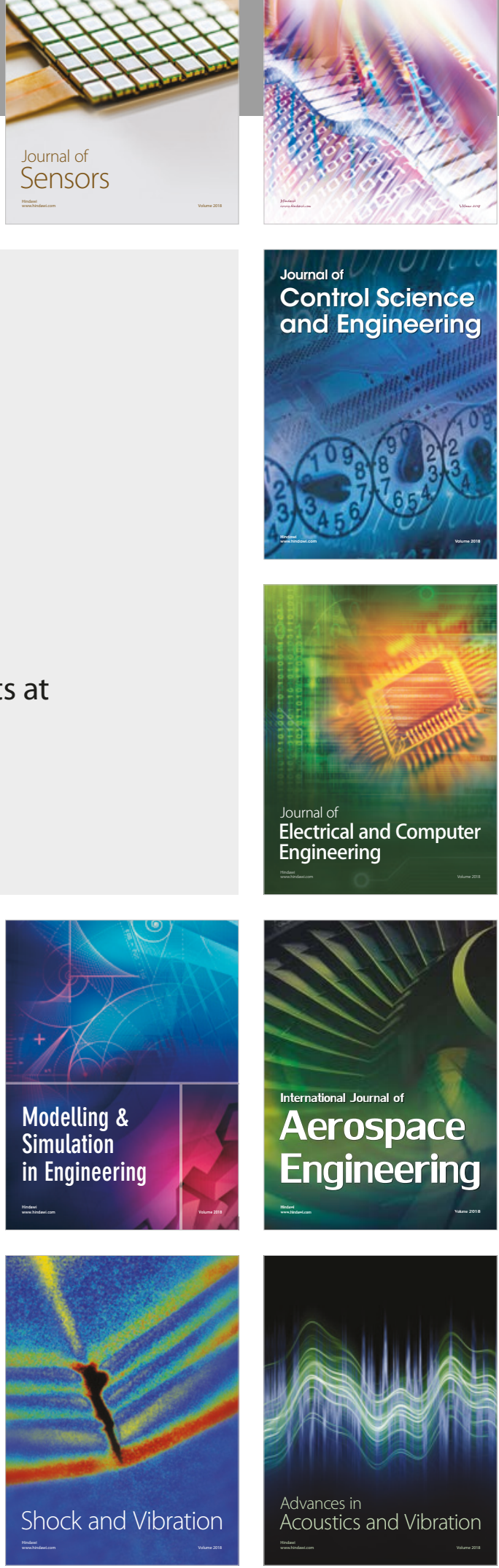